\title{
On Uniform Convergence of Sequences and Series of Fuzzy-Valued Functions
}

\author{
Ŭgur Kadak ${ }^{1,2}$ and Hakan Efe ${ }^{1}$ \\ ${ }^{1}$ Department of Mathematics, Faculty of Sciences, Gazi University, 06500 Ankara, Turkey \\ ${ }^{2}$ Department of Mathematics, Faculty of Sciences and Arts, Bozok University, 66100 Yozgat, Turkey \\ Correspondence should be addressed to Uğur Kadak; ugurkadak@gmail.com
}

Received 18 June 2014; Revised 26 August 2014; Accepted 10 September 2014

Academic Editor: Mahmut Işik

Copyright (c) 2015 U. Kadak and H. Efe. This is an open access article distributed under the Creative Commons Attribution License, which permits unrestricted use, distribution, and reproduction in any medium, provided the original work is properly cited.

\begin{abstract}
The class of membership functions is restricted to trapezoidal one, as it is general enough and widely used. In the present paper since the utilization of Zadeh's extension principle is quite difficult in practice, we prefer the idea of level sets in order to construct for a fuzzy-valued function via related trapezoidal membership function. We derive uniform convergence of fuzzy-valued function sequences and series with some illustrated examples. Also we study Hukuhara differentiation and Henstock integration of a fuzzyvalued function with some necessary inclusions. Furthermore, we introduce the power series with fuzzy coefficients and define the radius of convergence of power series. Finally, by using the notions of $\mathrm{H}$-differentiation and radius of convergence we examine the relationship between term by term $\mathrm{H}$-differentiation and uniform convergence of fuzzy-valued function series.
\end{abstract}

\section{Introduction}

The term uniform convergence was probably first used by Christoph Gudermann, in an 1838 paper on elliptic functions, where he employed the phrase "convergence in a uniform way" when the "mode of convergence" of a series is independent of two variables. While he thought it a "remarkable fact" when a series converged in this way, he did not give a formal definition or use the property in any of his proofs [1]. Later Karl Weierstrass, who attended his course on elliptic functions in 1839-1840, coined the term uniformly convergent which he used in his 1841 paper Zur Theorie der Potenzreihen, published in 1894. Independently a similar concept was used by Imre [2] and G. Stokes but without having any major impact on further development.

Due to the rapid development of the fuzzy logic theory, however, some of these basic concepts have been modified and improved. One of them is in the form of interval valued fuzzy sets. To achieve this we need to promote the idea of the level sets of fuzzy numbers and the related formulation of a representation of an interval valued fuzzy set in terms of its level sets. Once having the structure we then can supply the required extension to interval valued fuzzy sets.
The effectiveness of level sets is based on not only their required storage capacity but also their two-valued nature. Also the definition of these sets offers some advantages over the related membership functions.

Many authors have developed the different cases of sequence sets with fuzzy metric on a large scale. Başarir [3] has recently promoted some new sets of sequences of fuzzy numbers generated by a nonnegative regular matrix $A$, some of which reduced to Maddox's spaces $\ell_{\infty}(p ; F), c(p ; F)$, $c_{0}(p ; F)$, and $\ell(p ; F)$ for the special cases of that matrix $A$. Quite recently, Talo and Başar [4] have developed the main results of Başar and Altay [5] to fuzzy numbers and defined the alpha-, beta-, and gamma-duals and introduced the duals of these sets together with the classes of infinite matrices of fuzzy numbers mapping one of the classical set into another one. Also, Kadak and Ozluk [6] have introduced some new sets of sequences of fuzzy numbers with respect to the partial metric.

The rest of this paper is organized as follows. In Section 2, we give some necessary definitions and propositions related to the fuzzy numbers, sequences, and series of fuzzy numbers. We also report the most relevant and recent literature in this section. In Section 3, first the definition of fuzzy-valued 
function is given which will be used in the proof of our main results. In this section, generalized Hukuhara differentiation and Henstock integration are presented according to fuzzyvalued functions depending on real values $x$ and $t$. The final section is completed with the concentration of the results on uniform convergence of fuzzy-valued sequences and series. Also we examine the relationship between the radius of convergence of power series and the notion of uniform convergence with respect to fuzzy-valued function.

\section{Preliminaries, Background, and Notation}

A fuzzy number is a fuzzy set on the real axis, that is, a mapping $u: \mathbb{R} \rightarrow[0,1]$ which satisfies the following four conditions.

(i) $u$ is normal; that is, there exists an $x_{0} \in \mathbb{R}$ such that $u\left(x_{0}\right)=1$.

(ii) $u$ is fuzzy convex; that is, $u[\lambda x+(1-\lambda) y] \geq$ $\min \{u(x), u(y)\}$ for all $x, y \in \mathbb{R}$ and for all $\lambda \in[0,1]$.

(iii) $u$ is upper semicontinuous.

(iv) The set $[u]_{0}=\overline{\{x \in \mathbb{R}: u(x)>0\}}$ is compact (cf. Zadeh [7]), where $\overline{\{x \in \mathbb{R}: u(x)>0\}}$ denotes the closure of the set $\{x \in \mathbb{R}: u(x)>0\}$ in the usual topology of $\mathbb{R}$.

We denote the set of all fuzzy numbers on $\mathbb{R}$ by $E^{1}$ and called it the space of fuzzy numbers and the $\lambda$-level set $[u]_{\lambda}$ of $u \in E^{1}$ is defined by

$$
[u]_{\lambda}:= \begin{cases}\{t \in \mathbb{R}: u(t) \geq \lambda\}, & 0<\lambda \leq 1, \\ \overline{\{t \in \mathbb{R}: u(t)>\lambda\},} & \lambda=0 .\end{cases}
$$

The set $[u]_{\lambda}$ is closed, bounded, and nonempty interval for each $\lambda \in[0,1]$ which is defined by $[u]_{\lambda}:=\left[u^{-}(\lambda), u^{+}(\lambda)\right]$.

Theorem 1 (representation theorem [8]). Let $[u]_{\lambda}=$ $\left[u^{-}(\lambda), u^{+}(\lambda)\right]$ for $u \in E^{1}$ and for each $\lambda \in[0,1]$. Then the following statements hold.

(i) $u^{-}$is a bounded and nondecreasing left continuous function on $] 0,1]$.

(ii) $u^{+}$is a bounded and nonincreasing left continuous function on $] 0,1]$.

(iii) The functions $u^{-}$and $u^{+}$are right continuous at the point $\lambda=0$.

(iv) $u^{-}(1) \leq u^{+}(1)$.

Otherwise, if the pair of functions $u^{-}$and $u^{+}$holds the conditions (i)-(iv), then there exists a unique element $u \in E^{1}$ such that $[u]_{\lambda}:=\left[u^{-}(\lambda), u^{+}(\lambda)\right]$ for each $\lambda \in[0,1]$.

A fuzzy number is a convex fuzzy subset of $\mathbb{R}$ and is defined by its membership function. Let $u$ be a fuzzy number, whose membership function $\mu(x)$ can generally be defined as [9]

$$
\mu(x):= \begin{cases}\frac{x-u_{1}}{u_{2}-u_{1}}, & u_{1} \leq x \leq u_{2}, \\ 1, & u_{2} \leq x \leq u_{3}, \\ \frac{u_{4}-x}{u_{4}-u_{3}}, & u_{3} \leq x \leq u_{4}, \\ 0, & x<u_{1}, x>u_{4},\end{cases}
$$

where $u^{-}:\left[u_{1}, u_{2}\right] \rightarrow[0,1]$ and $u^{+}:\left[u_{3}, u_{4}\right] \rightarrow[0,1]$ are two strictly monotonical and continuous mappings from $\mathbb{R}$ to the closed interval $[0,1]$. If $\mu(x)$ is piecewise linear, then $u$ is referred to as a trapezoidal fuzzy number and is usually denoted by $u=\left(u_{1}, u_{2}, u_{3}, u_{4}\right)$. In particular, when $u_{2} \equiv u_{3}$, the trapezoidal form is reduced to a triangular form; that is, $u=\left(u_{1}, u_{2}, u_{4}\right)$. So, triangular forms are special cases of trapezoidal forms. Since $u^{-}$and $u^{+}$are both strictly monotonical and continuous functions, their inverse exists and should also be continuous and strictly monotonical.

Let $u, v, w \in E^{1}$ and $\alpha \in \mathbb{R}$. Then some algebraic operations, that is, level set addition, scalar multiplication, and product, are defined on $E^{1}$ by

$$
\begin{aligned}
u \oplus v=w \Longleftrightarrow[w]_{\lambda}=[u]_{\lambda} \oplus[v]_{\lambda} \\
\Longleftrightarrow w^{-}(\lambda)=u^{-}(\lambda)+v^{-}(\lambda), \\
\quad w^{+}(\lambda)=u^{+}(\lambda)+v^{+}(\lambda),
\end{aligned}
$$

$[\alpha u]_{\lambda}=\alpha[u]_{\lambda}$ and $u v=w \Leftrightarrow[w]_{\lambda}=[u]_{\lambda}[v]_{\lambda}$ for all $\lambda \in[0,1]$, where

$$
\begin{array}{r}
w^{-}(\lambda)=\min \left\{u^{-}(\lambda) v^{-}(\lambda), u^{-}(\lambda) v^{+}(\lambda),\right. \\
\left.u^{+}(\lambda) v^{-}(\lambda), u^{+}(\lambda) v^{+}(\lambda)\right\}, \\
w^{+}(\lambda)=\max \left\{u^{-}(\lambda) v^{-}(\lambda), u^{-}(\lambda) v^{+}(\lambda),\right. \\
\left.u^{+}(\lambda) v^{-}(\lambda), u^{+}(\lambda) v^{+}(\lambda)\right\} .
\end{array}
$$

Let $W$ be the set of all closed bounded intervals $A$ of $\mathbb{R}$ with endpoints $\underline{A}$ and $\bar{A}$; that is, $A:=[\underline{A}, \bar{A}]$. Define the relation $d$ on $W$ by $d(A, B):=\max \{|\underline{A}-\underline{B}|,|\bar{A}-\bar{B}|\}$. Then it can easily be observed that $d$ is a metric on $W$ (cf. Diamond and Kloeden [10]) and $(W, d)$ is a complete metric space (cf. Nanda [11]). Now, we can give the metric $D$ on $E^{1}$ by means of the Hausdorff metric $d$ as

$$
\begin{aligned}
D(u, v) & :=\sup _{\lambda \in[0,1]} d\left([u]_{\lambda},[v]_{\lambda}\right) \\
& :=\sup _{\lambda \in[0,1]} \max \left\{\left|u^{-}(\lambda)-v^{-}(\lambda)\right|,\left|u^{+}(\lambda)-v^{+}(\lambda)\right|\right\} .
\end{aligned}
$$

It is trivial that

$$
\begin{aligned}
D(u, \overline{0}) & =\sup _{\lambda \in[0,1]} \max \left\{\left|u^{-}(\lambda)\right|,\left|u^{+}(\lambda)\right|\right\} \\
& =\max \left\{\left|u^{-}(0)\right|,\left|u^{+}(0)\right|\right\} .
\end{aligned}
$$


Proposition 2 (see [12]). Let $u, v, w, z \in E^{1}$ and $\alpha \in \mathbb{R}$. Then,

(i) $\left(E^{1}, D\right)$ is a complete metric space (cf. Puri and Ralescu [13]);

(ii) $D(\alpha u, \alpha v)=|\alpha| D(u, v)$;

(iii) $D(u \oplus v, w \oplus v)=D(u, w)$;

(iv) $D(u \oplus v, w \oplus z) \leq D(u, w)+D(v, z)$;

(v) $|D(u, \overline{0})-D(v, \overline{0})| \leq D(u, v) \leq D(u, \overline{0})+D(v, \overline{0})$.

Remark 3 (cf. [14]). Then the following remarks can be given.

(a) Obviously the sequence $\left(u_{n}\right) \in \omega(F)$ converges to a fuzzy number $u$ if and only if $\left\{u_{n}^{-}(\lambda)\right\}$ and $\left\{u_{n}^{+}(\lambda)\right\}$ converge uniformly to $u^{-}(\lambda)$ and $u^{+}(\lambda)$ on $[0,1]$, respectively.

(b) The boundedness of the sequence $\left(u_{n}\right) \in \omega(F)$ is equivalent to the fact that

$$
\sup _{n \in \mathbb{N}} D\left(u_{n}, \overline{0}\right)=\sup _{n \in \mathbb{N} \lambda \in[0,1]} \max \left\{\left|u_{n}^{-}(\lambda)\right|,\left|u_{n}^{+}(\lambda)\right|\right\}<\infty .
$$

If the sequence $\left(u_{k}\right) \in \omega(F)$ is bounded then the sequences of functions $\left\{u_{k}^{-}(\lambda)\right\}$ and $\left\{u_{k}^{+}(\lambda)\right\}$ are uniformly bounded in $[0,1]$.

Definition 4 (see [14]). Let $\left(u_{k}\right) \in \omega(F)$. Then the expression ${ }_{\oplus} \sum_{k} u_{k}$ is called a series of fuzzy numbers with the level summation $_{\oplus} \sum$. Define the sequence $\left(s_{n}\right)$ via $n$th partial level sum of the series by $s_{n}=u_{0} \oplus u_{1} \oplus u_{2} \oplus \cdots \oplus u_{n}$ for all $n \in \mathbb{N}$. If the sequence $\left(s_{n}\right)$ converges to a fuzzy number $u$, then we say that the series ${ }_{\oplus} \sum_{k} u_{k}$ of fuzzy numbers converges to $u$ and write ${ }_{\oplus} \sum_{k} u_{k}=u$ which implies that

$$
\lim _{n \rightarrow \infty} \sum_{k=0}^{n} u_{k}^{-}(\lambda)=u^{-}(\lambda), \quad \lim _{n \rightarrow \infty} \sum_{k=0}^{n} u_{k}^{+}(\lambda)=u^{+}(\lambda),
$$

where the summation is in the sense of classical summation and converges uniformly in $\lambda \in[0,1]$.

2.1. Generalized Hukuhara Difference. A generalization of the Hukuhara difference proposed in [15] aims to overcome this situation.

Definition 5 (see [15, Definition 1]). The generalized Hukuhara difference $A \ominus B$ of two sets $A, B \in \mathscr{K}$ is defined as follows:

$$
A \ominus B=C \Longleftrightarrow\left\{\begin{array}{l}
A=B+C, \\
B=A+(-1) C .
\end{array}\right.
$$

Proposition 6 (see [16]). The following statements hold.

(a) If $A$ and $B$ are two closed intervals, then $D(A, B)=$ $D(A \ominus B,\{0\})$.

(b) Let $u:[a, b] \rightarrow I$ be such that $u(x)=\left[u^{-}(x), u^{+}(x)\right]$. Then, we have

$$
\begin{aligned}
\lim _{x \rightarrow x_{0}} u(x)=\ell & \Longleftrightarrow \lim _{x \rightarrow x_{0}}(u(x) \ominus \ell)=\{0\}, \\
\lim _{x \rightarrow x_{0}} u(x)=u\left(x_{0}\right) & \Longleftrightarrow \lim _{x \rightarrow x_{0}}\left(u(x) \ominus u\left(x_{0}\right)\right)=\{0\},
\end{aligned}
$$

where the limits are in the Hausdorff metric $d$ for intervals.

\section{Fuzzy-Valued Functions with the Level Sets}

Definition 7 (see [17]). Consider a fuzzy-valued function $u^{t}(x)$ from $\mathbb{R}$ into $E^{1}$ with respect to a membership function $\mu(x)$ which is called trapezoidal fuzzy number and is interpreted as follows:

$$
\mu(x)= \begin{cases}\frac{x-u_{1}(t)}{u_{2}(t)-u_{1}(t)}, & u_{1}(t) \leq x \leq u_{2}(t), \\ 1, & u_{2}(t) \leq x \leq u_{3}(t), \\ \frac{u_{4}(t)-x}{u_{4}(t)-u_{3}(t)}, & u_{3}(t) \leq x \leq u_{4}(t), \\ 0, & x<u_{1}(t), x>u_{4}(t) .\end{cases}
$$

Then, the pair of $u^{-}, u^{+}$depending on $t \in \mathbb{R}$ can be written as $u^{t}(x)=\left[\left(u_{2}(t)-u_{1}(t)\right) \lambda+u_{1}(t), u_{4}(t)-\left(u_{4}(t)-u_{3}(t)\right) \lambda\right] \in E^{1}$ for all $\lambda \in[0,1]$. Then, the function $u^{t}$ is said to be a fuzzyvalued function on $\mathbb{R}$.

Remark 8. The functions $u_{i}$ with $i \in\{1,2,3,4\}$ given in Definition 7 are also defined by $f_{i}(t)=k$ for all $t \in \mathbb{R}$ and the constant $k$.

Now, following Kadak [18] we give the classical sets $C_{F}[a, b]$ and $B_{F}[a, b]$ consisting of the continuous and bounded fuzzy-valued functions; that is,

$$
\begin{aligned}
C_{F}[a, b] & \\
:= & \left\{f^{t} \mid f^{t}:[a, b] \longrightarrow E^{1} \ni f^{t}\right. \\
& \text { continuous fuzzy-valued function } \\
& \forall x \in[a, b], t \in \mathbb{R}\}, \\
B_{F}[a, b] & \\
:= & \left\{f^{t} \mid f^{t}:[a, b] \longrightarrow E^{1} \ni f^{t}\right. \\
& \text { bounded fuzzy-valued function } \forall x, t \in[a, b]\} .
\end{aligned}
$$

Obviously, from Theorem 1, each function $f^{-}, f^{+}$is left continuous on $\lambda \in(0,1]$ and right continuous at $\lambda=0$. It was shown that $C_{F}[a, b]$ and $B_{F}[a, b]$ are complete with the metric $D_{F_{\infty}}$ as

$$
\begin{aligned}
D_{F_{\infty}}\left(f^{t}, g^{t}\right) & :=\sup _{x \in[a, b]}\left\{D\left(f^{t}(x), g^{t}(x)\right)\right\} \\
& =\sup _{x \in[a, b]}\left\{\sup _{\lambda \in[0,1]} d\left(\left[f^{t}(x)\right]_{\lambda},\left[g^{t}(x)\right]_{\lambda}\right)\right\} \\
& :=\max \left\{\sup _{\lambda \in[0,1] t \in[a, b]} \sup _{\lambda}\left|f_{\lambda}^{-}(t)-g_{\lambda}^{-}(t)\right|,\right.
\end{aligned}
$$


where $f^{t}=f^{t}(x)$ and $g^{t}=g^{t}(x)$ are the elements of the sets $C_{F}[a, b]$ or $B_{F}[a, b]$ with $x, t \in[a, b]$.

3.1. Generalized Hukuhara Differentiation and Henstock Integration. The notion of fuzzy differentiability comes from a generalization of the Hukuhara difference for compact convex sets. We prove several properties of the derivative of fuzzy-valued functions considered here. As a continuation of Hukuhara derivatives for real fuzzy-valued functions [19], we can define $\mathrm{H}$-differentiation of $f^{t}$ with respect to level sets.

Definition 9 (cf. [20]). A fuzzy-valued function $f^{t}: \mathbb{R} \rightarrow E^{1}$ is said to be generalized $\mathrm{H}$-differentiable with respect to the level sets at the point $x$ : if $\left(f^{t}\right)^{\prime}(x) \in E^{1}$ exists such that, for all $h>0$ sufficiently near to 0 , the $\mathrm{H}$-difference $f^{t}(x+h) \ominus f^{t}(x)$ exists then the H-derivative $\left(f^{t}\right)^{\prime}(x)$ is given as follows:

$$
\begin{aligned}
& \left(f^{t}\right)^{\prime}(x) \\
& =\lim _{h \rightarrow 0^{+}}\left[\frac{f^{t}(x+h) \ominus f^{t}(x)}{h}\right]_{\lambda} \\
& =\left[\lim _{h \rightarrow 0^{+}} \frac{f_{\lambda}^{-}(t+h)-f_{\lambda}^{-}(t)}{h}, \lim _{h \rightarrow 0^{+}} \frac{f_{\lambda}^{+}(t+h)-f_{\lambda}^{+}(t)}{h}\right] \\
& =\left[\left(f_{\lambda}^{-}(t)\right)^{\prime},\left(f_{\lambda}^{+}(t)\right)^{\prime}\right] .
\end{aligned}
$$

From here, we remind that the $\mathrm{H}$-derivative of $f^{t}$ at $x \in \mathbb{R}$ depends on $t$ and $\lambda$. Therefore, $f^{t}$ is $\mathrm{H}$-differentiable if and only if $f_{\lambda}^{-}$and $f_{\lambda}^{+}$are classical differentiable functions.

Definition 10 (see [21, Definition 8.7]). A fuzzy valued function $f^{t}$ is said to be fuzzy Henstock, in short FH-integrable if, for any $\epsilon>0$, there exists $\delta>0$ such that

$$
\begin{aligned}
D\left(\sum_{\oplus} \sum_{P}(v-u) f^{t}(\xi), I\right) & \\
=\sup _{\lambda \in[0,1]} \max & \left\{\left|\sum_{P}(v-u) f_{\lambda}^{-}(t)-I_{\lambda}^{-}\right|,\right. \\
& \left.\left|\sum_{P}(v-u) f_{\lambda}^{+}(t)-I_{\lambda}^{+}\right|\right\}<\epsilon
\end{aligned}
$$

for any division $P=\{[u, v] ; \xi\}$ of $[a, b]$ with the norms $\Delta(P)<$ $\delta$, where $I:=(\mathrm{FH}) \int_{a}^{b} f^{t}(x) d x$ and $f^{t}$ is also called $\mathrm{FH}$ integrable. One can conclude that $\sum_{P}$ in (15) denotes the usual Riemann sum for any division $P$ of $[a, b]$.

Theorem 11 (see [21, Theorem 8.8]). Let $f^{t} \in C_{F}[a, b]$ and let $f^{t}$ be a FH-integrable function. If there exists $x_{0} \in[a, b]$ such that $f_{\lambda}^{-}\left(x_{0}\right)=f_{\lambda}^{+}\left(x_{0}\right)=1$, then

$$
\left[(F H) \int_{a}^{b} f^{t}(x) d x\right]_{\lambda}=\left[\int_{a}^{x_{0}} f_{\lambda}^{-}(t) d t, \int_{x_{0}}^{b} f_{\lambda}^{+}(t) d t\right]
$$

Remark 12. We remark that the integrals on the right hand side of (16) exist in the usual sense for all $\lambda \in[0,1]$. It is easy to see that the pair of functions $f_{\lambda}^{ \pm}:[a, b] \rightarrow \mathbb{R}$ are continuous.

\section{Uniform Convergence of Fuzzy-Valued Functions}

Definition 13. Let $\left\{f_{n}^{t}(x)\right\}$ be a sequence of fuzzy-valued functions defined on a set $A \subseteq \mathbb{R}$ with respect to the sequence $t=\left(t_{n}\right)$ with real or complex terms. We say that $\left\{f_{n}^{t}(x)\right\}$ converges pointwise on $A$ if for each $x \in A$ the sequence $\left\{f_{n}^{t}(x)\right\}$ converges for all $x \in A$. If a sequence $\left\{f_{n}^{t}(x)\right\}$ converges pointwise, then we can define a fuzzyvalued function $u^{t}: A \rightarrow E^{1}$ by

$$
\lim _{n \rightarrow \infty} f_{n}^{t}(x)=u^{t}(x) \quad \text { for each } x \in A, n \in \mathbb{N} .
$$

On the other hand, $\left\{f_{n}^{t}(x)\right\}$ converges to $u^{t}$ on $A$ if and only if, for each $x \in A$ and for an arbitrary $\epsilon>0$, there exists an integer $N=N(\epsilon, x)$ such that $D\left(f_{n}^{t}(x), u^{t}(x)\right)<\epsilon$ whenever $n>N$. The integer $N$ in the definition of pointwise convergence may, in general, depend on both $\epsilon>0$ and $x \in A$. If, however, one integer can be found that works for all points in $A$, then the convergence is said to be uniform. That is, a sequence of fuzzy-valued functions $\left\{f_{n}^{t}(x)\right\}$ converges uniformly to $u^{t}$ if, for each $\epsilon>0$, there exists an integer $N(\epsilon)$ such that

$$
D\left(f_{n}^{t}(x), u^{t}(x)\right)<\epsilon \quad \text { whenever } n>N(\epsilon), \forall x \in A .
$$

Obviously the sequence $\left(f_{n}^{t}\right)$ of fuzzy-valued functions converges (uniformly) to a fuzzy valued-function $u^{t}$ if and only if $\left\{\left(f_{n}^{t}\right)^{-}(\lambda)\right\}$ and $\left\{\left(f_{n}^{t}\right)^{+}(\lambda)\right\}$ converge uniformly to $\left\{\left(u^{t}\right)^{-}(\lambda)\right\}$ and $\left\{\left(u^{t}\right)^{+}(\lambda)\right\}$ in $\lambda \in[0,1]$, respectively. On the other hand $\left(f_{n}^{t}\right)$ converges (uniformly) to $u^{t}$ if and only if the sequence $t_{n}$ converges to a real (complex) number $t$.

Example 14. Consider a sequence $f_{n}^{t}=\left(t_{n}, u_{1}, u_{2}, u_{3}\right)$ of fuzzy-valued functions from $\left[t_{n}, u_{3}\right]$ into $E^{1}$ where $\left(t_{n}\right) \in \omega$, whose membership function $\mu(x)$ is defined as

$$
\mu(x)= \begin{cases}\frac{x-t_{n}}{u_{1}-t_{n}}, & t_{n} \leq x \leq u_{1}, \\ 1, & u_{1} \leq x \leq u_{2}, \\ \frac{u_{3}-x}{u_{3}-u_{2}}, & u_{2} \leq x \leq u_{3}, \\ 0, & x<t_{n}, x>u_{3},\end{cases}
$$

where $\max \left\{t_{n}\right\} \leq u_{1}$ for all $n \in \mathbb{N}$. Then, the membership function can be written as

$$
\begin{aligned}
{\left[f_{n}^{t}\right]_{\lambda} } & =\left[\left(f_{n}^{t}\right)^{-}(\lambda),\left(f_{n}^{t}\right)^{+}(\lambda)\right] \\
& =\left[\left(u_{1}-t_{n}\right) \lambda+t_{n}, u_{3}-\left(u_{3}-u_{2}\right) \lambda\right]
\end{aligned}
$$

consisting of each function $f^{-}, f^{+}$depending on $t$ and $\lambda \epsilon$ $[0,1]$. Suppose that the sequence $\left(t_{n}\right)$ converges; then $\left\{f_{n}^{t}\right\}$ 
converges uniformly to the fuzzy-valued function $u^{t}$ which is given by

$$
\mu(x)= \begin{cases}\frac{x-t}{u_{1}-t}, & t \leq x \leq u_{1} \\ 1, & u_{1} \leq x \leq u_{2} \\ \frac{u_{3}-x}{u_{3}-u_{2}}, & u_{2} \leq x \leq u_{3} \\ 0, & x<t, x>u_{3} .\end{cases}
$$

In this form one can easily conclude that $t_{n} \rightarrow t$ if and only if $\left(f_{n}^{t}\right)^{-}(\lambda) \rightarrow\left(u^{t}\right)^{-}(\lambda)$ uniformly in $\lambda \in[0,1]$.

Remark 15. If the sequence $\left(t_{n}\right)$ is, respectively, replaced by each of the fix numbers $u_{1}, u_{2}$, and $u_{3}$ in Example 14, that is, $f_{n}^{t}=\left(u_{1}, t_{n}, u_{2}, u_{3}\right)$ where $t_{n} \in\left[u_{1}, u_{2}\right]$ for all $n \in \mathbb{N}$, then $f_{n}^{t} \rightarrow u^{t}$ uniformly if and only if $t_{n} \rightarrow t$ or $\left(f_{n}^{t}\right)^{-}(\lambda) \rightarrow$ $\left(u^{t}\right)^{-}(\lambda)$ uniformly in $\lambda \in[0,1]$ where $u^{t}=\left(u_{1}, t, u_{2}, u_{3}\right)$. Similarly we take $f_{n}^{t}=\left(u_{1}, u_{2}, t_{n}, u_{3}\right)$ where $t_{n} \in\left[u_{2}, u_{3}\right]$ for all $n \in \mathbb{N}$ and then $f_{n}^{t} \rightarrow u^{t}$ uniformly if and only if $t_{n} \rightarrow t$ for all $t \in \mathbb{R}$, or $\left(f_{n}^{t}\right)^{+}(\lambda) \rightarrow\left(u^{t}\right)^{+}(\lambda)$ uniformly for each $\lambda \in[0,1]$ where $u_{t}=\left(u_{1}, u_{2}, t, u_{3}\right)$.

Now, in the following example, we give some cases of membership functions with two constants.

Example 16. Consider a sequence $f_{n}^{t}=\left(u_{1}, t_{n}, t_{n}+k, u_{4}\right)$ of fuzzy-valued functions from $\left[u_{1}, u_{4}\right]$ into $E^{1}$ for the constant $k$, whose membership function $\mu(x)$ is defined as

$$
\mu(x)= \begin{cases}\frac{x-u_{1}}{t_{n}-u_{1}}, & u_{1} \leq x \leq t_{n}, \\ 1, & t_{n} \leq x \leq t_{n}+k, \\ \frac{u_{4}-x}{u_{4}-t_{n}-k}, & t_{n}+k \leq x \leq u_{4}, \\ 0, & x<u_{1}, x>u_{4},\end{cases}
$$

where $u_{1} \leq \min \left\{t_{n}\right\}$ and $u_{4} \geq \max \left\{t_{n}+k\right\}$ for all $n \in \mathbb{N}$ and the constant $k$. Then, it is obvious that

$$
\left[f_{n}^{t}\right]_{\lambda}=\left[\left(t_{n}-u_{1}\right) \lambda+u_{1}, u_{4}-\left(u_{4}-t_{n}-k\right) \lambda\right]
$$

for all $\lambda \in[0,1]$. Hence, as we have seen above $t_{n} \rightarrow t$ if and only if $\left(f_{n}^{t}\right)^{+}(\lambda) \rightarrow\left(u^{t}\right)^{+}(\lambda)$ and $\left(f_{n}^{t}\right)^{-}(\lambda) \rightarrow\left(u^{t}\right)^{-}(\lambda)$ uniformly in $\lambda \in[0,1]$ where

$$
\begin{aligned}
{\left[u^{t}\right]_{\lambda} } & =\left[\left(u^{t}\right)^{-}(\lambda),\left(u^{t}\right)^{+}(\lambda)\right] \\
& =\left[\left(t-u_{1}\right) \lambda+u_{1}, u_{4}-\lambda\left(u_{4}-t-k\right)\right],
\end{aligned}
$$

$u_{1} \leq t$ and $u_{4} \geq t+k$ for all $t \in \mathbb{R}$ and the constant $k$. Therefore, we derive that $f_{n}^{t} \rightarrow u^{t}$ converges (uniformly).

Remark 17. If $t_{n}$ and $t_{n}+k$ are, respectively, replaced by each of the fixed numbers $u_{i}$ with $i \in\{1,2,3,4\}$ given in Example 16, that is, $f_{n}^{t}=\left(u_{1}, u_{2}, t_{n}, t_{n}+k\right)$ where $u_{2} \leq \min \left\{t_{n}\right\}$ for all $n \in \mathbb{N}$ and a constant $k$, then $f_{n}^{t} \rightarrow u^{t}$ uniformly if and only if $t_{n} \rightarrow t$ or $\left(f_{n}^{t}\right)^{+}(\lambda) \rightarrow\left(u^{t}\right)^{+}(\lambda)$ uniformly in $\lambda \in[0,1]$ where $u_{t}=\left(u_{1}, u_{2}, t, t+k\right)$. Other cases with two constants can be obtained similarly.
Example 18. Consider a sequence $f_{n}^{t}=\left(u_{1}, t_{n}, t_{n}+k, t_{n}+L\right)$ of fuzzy-valued functions for the constants $k, L$ with $L \geq k$, whose membership function $\mu(x)$ is defined:

$$
\mu_{f_{n}^{t}}(x)= \begin{cases}\frac{x-u_{1}}{t_{n}-u_{1}}, & u_{1} \leq x \leq t_{n}, \\ \frac{t_{n}+L-x}{L-k}, & t_{n} \leq x \leq t_{n}+k \\ 0, & x<u_{1}, x>t_{n}+L,\end{cases}
$$

where $u_{1} \leq \min \left\{t_{n}\right\}$ for all $n \in \mathbb{N}$. Then, it is clear that $\left[f_{n}^{t}\right]_{\lambda}=$ $\left[\left(t_{n}-u_{1}\right) \lambda+u_{1}, t_{n}+L-\lambda(L-k)\right]$ for all $\lambda \in[0,1]$. Then $t_{n} \rightarrow t$ if and only if $\left(f_{n}^{t}\right)^{+}(\lambda) \rightarrow\left(u^{t}\right)^{+}(\lambda)$ and $\left(f_{n}^{t}\right)^{-}(\lambda) \rightarrow\left(u^{t}\right)^{-}(\lambda)$ uniformly in $\lambda \in[0,1]$ where

$$
\left[u^{t}\right]_{\lambda}=\left[\left(t-u_{1}\right) \lambda+u_{1},(t+L)-\lambda(L-k)\right], \quad\left(u_{1} \leq t\right) .
$$

Remark 19. If we take $u_{1}$ as a function based on $t$ in Example 18, that is, $f_{n}^{t}=\left(t_{n}, t_{n}+k_{1}, t_{n}+k_{2}, t_{n}+k_{3}\right)$ where the constants $k_{1} \leq k_{2} \leq k_{3}$, then $f_{n}^{t} \rightarrow u^{t}$ uniformly if and only if $t_{n} \rightarrow t$ or $\left(f_{n}^{t}\right)^{+}(\lambda) \rightarrow\left(u^{t}\right)^{+}(\lambda)$ uniformly in $\lambda \in[0,1]$ where $u_{t}=\left(t, t+k_{1}, t+k_{2}, t+k_{3}\right)$.

Theorem 20 (see [17]). Then, the following statements are valid.

(i) A sequence offuzzy-valued functions $\left\{\left(f_{t}\right)_{n}(x)\right\}$ defined on a set $A \subseteq \mathbb{R}$ converges uniformly to a fuzzy-valued function $f^{t}$ on $A$ if and only if

$$
\begin{array}{r}
\delta_{n}=\sup _{x \in A} D\left[\left(f_{t}\right)_{n}(x), u_{t}(x)\right] \\
=\sup _{x \in A}\left\{\sup _{\lambda \in[0,1]} d\left(\left[\left(f_{t}\right)_{n}(x)\right]_{\lambda},\left[u_{t}(x)\right]_{\lambda}\right)\right\} \\
\text { with } \lim _{n \rightarrow \infty} \delta_{n}=\overline{0 .}
\end{array}
$$

(ii) The limit of a uniformly convergent sequence of continuous fuzzy-valued functions $\left\{\left(f_{t}\right)_{n}\right\}$ on a set $A$ is continuous. That is, for each $a \in A$,

$$
\lim _{x \rightarrow a}\left[\lim _{n \rightarrow \infty} f_{n}^{t}(x)\right]=\lim _{n \rightarrow \infty}\left[\lim _{x \rightarrow a}\left(f_{t}\right)_{n}(x)\right] .
$$

Theorem 21. A sequence of fuzzy-valued functions $\left\{\left(f_{t}\right)_{k}\right\}$ defined on a set A converges uniformly if and only if it is uniformly Cauchy; that is, for an arbitrary $\varepsilon>0$ there is a number $N=N(\varepsilon)$ such that

$$
D\left(f_{m}^{t}(x), f_{n}^{t}(x)\right)<\varepsilon \quad \text { whenever } m>n>N(\varepsilon), \forall x \in A,
$$

or equivalently, $\sup _{x \in A} D\left(f_{m}^{t}(x), f_{n}^{t}(x)\right)<\varepsilon$. 
Theorem 22 (cf. [17]). Suppose that $f_{n}^{t}(x) \in C_{F}[a, b]$ for all $n \in \mathbb{N}$ such that $\left\{f_{n}^{t}(x)\right\}$ converges uniformly to $f^{t}(x)$. By combining this and inclusion (28), the equalities

$$
\begin{aligned}
\lim _{n \rightarrow \infty}\left[(F H) \int_{a}^{b} f_{n}^{t}(x) d x\right]_{\lambda} \\
=\left[(F H) \int_{a}^{b} \lim _{n \rightarrow \infty} f_{n}^{t}(x) d x\right]_{\lambda} \\
=\left[(F H) \int_{a}^{b} f^{t}(x) d x\right]_{\lambda}=\left[\int_{a}^{p} f_{\lambda}^{-}(t) d t, \int_{p}^{b} f_{\lambda}^{+}(t) d t\right]
\end{aligned}
$$

hold for $f_{1}^{-}=f_{1}^{+}=p$ where the integral (FH) $\int_{a}^{b} f^{t}(x) d x$ exists for all $x, t \in[a, b]$ and $\lambda \in[0,1]$.

Theorem 23. Suppose that $\left\{f_{n}^{t}\right\}$ is a sequence of FH-integrable functions defined on a closed interval $[a, b]$. If $f_{n}^{t} \rightarrow f^{t}$ uniformly on $[a, b]$, then $f^{t}$ is FH-integrable on $[a, b]$, and

$$
\lim _{n \rightarrow \infty}\left[(F H) \int_{a}^{b} f_{n}^{t}(x) d x\right]_{\lambda}=\left[(F H) \int_{a}^{b} f^{t}(x) d x\right]_{\lambda} .
$$

Also, for each $r \in[a, b]$, then

$$
\left[(F H) \int_{a}^{r} f_{n}^{t}(x) d x\right]_{\lambda} \rightarrow\left[(F H) \int_{a}^{r} f^{t}(x) d x\right]_{\lambda}
$$

uniformly in $\lambda \in[0,1]$.

Proof. By taking into account Theorem 22, we need only to show that the limit function $f^{t}$ is $\mathrm{FH}$-integrable on $[a, b]$. We see that $\left\{f_{n}^{t}\right\}$ is bounded, because each $\left\{f_{n}^{t}\right\}$ is FH-integrable on $[a, b]$. Also, the limit function $f^{t}$ is bounded, since, using triangle inequality and Theorem 20 , we have

$$
\begin{aligned}
D\left(f^{t}(x), \overline{0}\right) \leq & D\left(f_{n}^{t}(x), f^{t}(x)\right) \\
& +D\left(f_{n}^{t}(x), \overline{0}\right) \leq \delta_{n}+D\left(f_{n}^{t}(x), \overline{0}\right),
\end{aligned}
$$

where $\delta_{n} \in c_{0}(F)$. Besides this, $f_{n}^{t} \rightarrow f^{t}$ uniformly on $[a, b]$, given any $\varepsilon>0$, there exists an integer $N(\varepsilon)$ such that

$$
\begin{gathered}
D\left(f_{n}^{t}(x), f^{t}(x)\right)<\frac{\varepsilon}{3(b-a)} \\
\forall x, t \in[a, b], \quad \lambda \in[0,1],
\end{gathered}
$$

for $n \geq N(\varepsilon)$. Since $\left\{f_{N}^{t}\right\}$ is FH-integrable, there exists a partition $P$ of $[a, b]$ such that

$$
U\left(P, f_{N}^{t}\right)-L\left(P, f_{N}^{t}\right)<\frac{\varepsilon}{3} .
$$

For each $x \in[a, b]$, using the inclusion (34) with $n=N$ implies that

$$
f_{N}(t)_{\lambda}^{ \pm}-\frac{\varepsilon}{3(b-a)}<f(t)_{\lambda}^{ \pm}<f_{N}(t)_{\lambda}^{ \pm}+\frac{\varepsilon}{3(b-a)}
$$

and therefore,

$$
L\left(P, f_{N}^{t}\right)-\frac{\varepsilon}{3}<L\left(P, f^{t}\right) \leq U\left(P, f^{t}\right)<U\left(P, f_{N}^{t}\right)+\frac{\varepsilon}{3} .
$$

Consequently,

$$
\begin{aligned}
U\left(P, f^{t}\right)-L\left(P, f^{t}\right)< & U\left(P, f_{N}^{t}\right)-L\left(P, f_{N}^{t}\right) \\
& +\frac{2 \varepsilon}{3}<\frac{\varepsilon}{3}+\frac{2 \varepsilon}{3}=\varepsilon
\end{aligned}
$$

showing that $f^{t}$ is FH-integrable on $[a, b]$. Finally, for $n \geq N$ and for each $r \in[a, b],(34)$ implies that

$$
\begin{aligned}
& D_{\infty}\left((\mathrm{FH}) \int_{a}^{r} f_{n}^{t}(x) d x,(\mathrm{FH}) \int_{a}^{r} f^{t}(x) d x\right) \\
& =\sup _{x \in[a, b]} d\left(\left[(\mathrm{FH}) \int_{a}^{r} f_{n}^{t}(x) d x\right]_{\lambda},\left[(\mathrm{FH}) \int_{a}^{r} f^{t}(x) d x\right]_{\lambda}\right) \\
& \quad \leq \int_{a}^{r} \sup _{t \in[a, b] \lambda \in[0,1]}\left\{\operatorname { m a x } \left\{\left|\left(f_{n}^{t}\right)_{\lambda}^{-}-\left(f^{t}\right)_{\lambda}^{-}\right|,\right.\right. \\
& \left.\left.\quad<\left(f_{n}^{t}\right)_{\lambda}^{+}-f(t)_{\lambda}^{+} \mid\right\}\right\} d t \\
& <\frac{\varepsilon(r-a)}{3(b-a)} \leq \frac{\varepsilon(b-a)}{3(b-a)}=\frac{\varepsilon}{3}
\end{aligned}
$$

and the proof is complete.

Theorem 24. Consider $\left\{f_{n}^{t}\right\}$ is a sequence of fuzzy-valued functions such that

(i) $\left\{f_{n}^{t}\right\} \in C_{F}^{1}[a, b]$;

(ii) there exists a point $x_{0} \in[a, b]$ such that $\left\{f_{n}^{t}\left(x_{0}\right)\right\}$ converges;

(iii) $\left(f_{n}^{t}\right)^{\prime} \rightarrow g^{t}$ uniformly on $[a, b]$.

Then $\left\{f_{n}^{t}\right\}$ converges uniformly to some $f^{t}$ on $[a, b]$ such that $\left(f^{t}\right)^{\prime}(x)=g^{t}(x)$ on $[a, b]$.

Proof. By (iii), $\left\{\left(f_{n}^{t}\right)^{\prime}\right\}$ is uniformly convergent to $g^{t}$ on any closed interval contained in $[a, b]$, say in an interval with endpoints $x_{0}$ and $x \in[a, b]$. By using Theorem 22, we have

$$
\begin{aligned}
& {\left[\text { (FH) } \int_{x_{0}}^{x} g(y) d y\right]_{\lambda}} \\
& \quad=\lim _{n \rightarrow \infty}\left[(\mathrm{FH}) \int_{x_{0}}^{x}\left(f_{n}^{t}\right)^{\prime}(y) d y\right]_{\lambda} \\
& =\lim _{n \rightarrow \infty}\left[f_{n}^{t}(x)-f_{n}^{t}\left(x_{0}\right)\right]_{\lambda} \\
& =\left[\lim _{n \rightarrow \infty}\left(\left(f_{n}^{t}\right)_{\lambda}^{-}-\left(f_{n}^{t_{0}}\right)_{\lambda}^{-}\right), \lim _{n \rightarrow \infty}\left(\left(f_{n}^{t}\right)_{\lambda}^{+}-\left(f_{n}^{t_{0}}\right)_{\lambda}^{+}\right)\right],
\end{aligned}
$$


for all $t$ and $t_{0}$ belonging to $[a, b]$ by the fundamental theorem of calculus, and the convergence is uniform on $[a, b]$. Since $\lim _{n} f_{n}^{t}\left(x_{0}\right)$ exists by (ii), we can add this term to both sides and obtain

$$
\lim _{n \rightarrow \infty} f_{n}^{t}(x)=\left[(\mathrm{FH}) \int_{x_{0}}^{x} g(y) d y\right]_{\lambda}+\left[\lim _{n \rightarrow \infty} f_{n}^{t}\left(x_{0}\right)\right]_{\lambda}
$$

and the convergence is uniform on $[a, b]$. We may now take $f^{t}(x)=\lim _{n \rightarrow \infty} f_{n}^{t}(x)$, and the last equation holds

$$
f^{t}(x)=\left[(\mathrm{FH}) \int_{x_{0}}^{x} g(y) d y\right]_{\lambda}+\left[\lim _{n \rightarrow \infty} f_{n}^{t}\left(x_{0}\right)\right]_{\lambda} .
$$

Now, $g^{t}$, being the fuzzy limit on $[a, b]$, is continuous on $[a, b]$, and so $G^{t}(x)=\left[(\mathrm{FH}) \int_{x_{0}}^{x} g(y) d y\right]_{\lambda}$ is H-differentiable and $\left(G_{n}^{t}\right)^{\prime}(x)=g^{t}(x)$ on $[a, b]$. Therefore, the last inequality and Definition 9 imply that

$$
\left(f^{t}\right)^{\prime}(x)=g^{t}(x),\left(f^{t}\right)^{\prime}(x)=\lim _{n \rightarrow \infty}\left(f_{n}^{t}\right)^{\prime}(x)
$$

for all $x, t \in[a, b]$ and $\lambda \in[0,1]$. This completes the proof.

Theorem 24 continues to hold under a weaker hypothesis. However, we cannot replace the third condition, namely, the uniform convergence of the sequence $\left(f_{n}^{t}\right)^{\prime}$, with pointwise convergence. Now we state an improved version of Theorem 24 without its proof.

Theorem 25. Suppose that $\left\{f_{n}^{t}\right\}$ is a sequence of fuzzy-valued functions such that

(i) each $\left\{f_{n}^{t}\right\}$ is H-differentiable on $[a, b]$;

(ii) there exists a point $x_{0} \in[a, b]$ such that $\left\{f_{n}^{t}\left(x_{0}\right)\right\}$ converges;

(iii) $\left(f_{n}^{t}\right)^{\prime} \rightarrow g^{t}$ uniformly on $[a, b]$.

Then $\left\{f_{n}^{t}\right\}$ converges uniformly to some $f^{t}$ on $[a, b]$ such that $\left(f^{t}\right)^{\prime}(x)=g^{t}(x)$ on $[a, b]$.

\section{Uniform Convergence of Fuzzy-Valued Function Series}

Definition 13 suggests that we continue our discussion from sequences of fuzzy-valued functions to series of fuzzy-valued functions with the level sets. Consider a sequence of functions $\left\{f_{n}^{t}(x)\right\}$ defined on a set $A$. Recall that the level sum ${ }_{\oplus} \sum_{k=1}^{\infty} f_{k}^{t}$ is called a series of fuzzy-valued functions. Form a new sequence of partial level sums of functions $\left\{S_{n}^{t}(x)\right\}$ defined by

$$
S_{n}^{t}(x)=\sum_{\oplus=1}^{n} f_{k}^{t}(x), \quad(x \in A, t \in \mathbb{R}) .
$$

If the sequence $\left\{S_{n}^{t}(x)\right\}$ converges at a point $x \in A$, then we say that the series of fuzzy-valued functions converges at $x$. If the sequence $\left\{S_{n}^{t}(x)\right\}$ converges at all points of $A$, then we say that $\sum_{k=1}^{\infty} f_{k}^{t}$ converges (pointwise) on $A$ and write the level sum function as

$$
f^{t}(x): \lim _{n \rightarrow \infty} \sum_{k=1}^{n} f_{k}^{t}(x) .
$$

Definition 26. The series $\sum_{\oplus=1}^{\infty} f_{k}^{t}(x)$ is said to be uniformly convergent to a fuzzy-valued function $f^{t}(x)$ on $A$ if the partial level sum $\left\{S_{n}^{t}(x)\right\}$ converges uniformly to $f^{t}(x)$ on $A$. That is, the series converges uniformly to $f^{t}(x)$ if given any $\varepsilon>0$, there exists an integer $n_{0}(\varepsilon)$ such that

$$
\begin{gathered}
D\left(\sum_{\oplus_{k=1}}^{n} f_{k}^{t}(x), f^{t}(x)\right) \\
=\max \left\{\sup _{\lambda \in[0,1] t \in A} \sup _{t \in A}\left|\sum_{k=1}^{n} f_{k}(t)_{\lambda}^{-}-f(t)_{\lambda}^{-}\right|,\right. \\
\left.\sup _{\lambda \in[0,1]} \sup _{t \in A}\left|\sum_{k=1}^{n} f_{k}(t)_{\lambda}^{+}-f(t)_{\lambda}^{+}\right|\right\}<\varepsilon
\end{gathered}
$$

whenever $n \geq n_{0}(\varepsilon)$.

Corollary 27. If each $\left\{f_{k}^{t}\right\}$ is a continuous fuzzy-valued function on $A \subseteq \mathbb{R}$ for each $k \geq 1$ and if ${ }_{\oplus} \sum_{k \geq 1} f_{k}^{t}(x)$ is uniformly convergent to $f^{t}(x)$ on $A$, then $f^{t}$ must be continuous on $A$ for all $x, t \in A$.

Corollary 28. A series ${ }_{\oplus} \sum_{k=1}^{\infty} f_{k}^{t}(x)$ converges uniformly on $a$ set $A$ if and only if the sequence of partial level sums is uniformly Cauchy on $A$; that is, for an arbitrary $\varepsilon>0$ there is a number $N=N(\varepsilon)$ such that

$$
\begin{aligned}
D & \left(S_{m}^{t}(x), S_{n}^{t}(x)\right) \\
& =D\left(\sum_{k=n+1}^{m} f_{k}^{t}(x), \overline{0}\right) \\
& =\max \left\{\sup _{t \in A} \sup _{\lambda \in[0,1]}\left|\sum_{k=n+1}^{m} f_{k}(t)_{\lambda}^{-}\right|,\right. \\
& \left.\sup _{t \in A} \sup _{\lambda \in[0,1]}\left|\sum_{k=n+1}^{m} f_{k}(t)_{\lambda}^{+}\right|\right\}<\varepsilon,
\end{aligned}
$$

whenever $m>n \geq n_{0}(\varepsilon)$.

Corollary 29. Suppose that $\left\{f_{k}^{t}(x)\right\}$ is a sequence in $C_{F}[a, b]$ and that ${ }_{\oplus} \sum_{k=0}^{\infty} f_{k}^{t}(x)$ converges uniformly to $f^{t}(x)$ on $[a, b]$. Then,

$$
\begin{aligned}
{[(F H)} & \left.\sum_{\oplus=0}^{\infty} \int_{a}^{b} f_{k}^{t}(x) d x\right]_{\lambda} \\
& =\left[(F H) \int_{a}^{b} \sum_{k=0}^{\infty} f_{k}^{t}(x) d x\right]_{\lambda} \\
& =\left[(F H) \int_{a}^{b} f^{t}(x) d x\right]_{\lambda},
\end{aligned}
$$

where $(F H) \int_{a}^{b} f^{t}(x) d x$ exists for all $x, t \in[a, b]$ and $\lambda \in[0,1]$. 
Corollary 28 shows that an analogue of the comparison test, namely, a sufficient condition for the uniform convergence of a fuzzy-valued function series. Indeed, the following result is a simple and direct test for the uniform convergence of these series.

Theorem 30. Let ${ }_{\oplus} \sum_{k=0}^{\infty} f_{k}^{t}(x)$ be a series of fuzzy-valued functions on a subset $A$ of $\mathbb{R}$. Consider there exists a convergent series $\sum_{k=1}^{\infty} M_{k}$ of nonnegative real numbers such that for all $k \in \mathbb{N}$ and $x \in A$ we have

$$
\max \left\{\sup _{t \in A} \sup _{\lambda \in[0,1]}\left|\left(f_{k}^{t}\right)^{-}(\lambda)\right|, \sup _{t \in A} \sup _{\lambda \in[0,1]}\left|\left(f_{k}^{t}\right)^{+}(\lambda)\right|\right\} \leq M_{k}
$$

Then $\sum_{k=0}^{\infty} f_{k}^{t}(x)$ converges uniformly.

Proof. Suppose that $\sum_{\oplus=0}^{\infty} f_{k}^{t}(x)$ is dominated by a convergent series $\sum_{k=1}^{\infty} M_{k}$. That each of the series $\sum_{k=0}^{\infty}\left|\left(f_{k}^{t}\right)^{ \pm}(\lambda)\right|$ converges on $A$ comes from the comparison test for real series. To verify the uniform convergence of $\sum_{k} f_{k}^{t}(x)$, take into account Cauchy criterion for the series $\sum_{k=1}^{\infty} M_{k}$. Hence, given $\epsilon>0$, there exists an integer $n_{0}=n_{0}(\epsilon)$ such that, for $m>n>n_{0}$, we have $\sum_{k=n+1}^{m} M_{k}<\epsilon$. For all $m>n>n_{0}$, we also obtain

$$
\begin{aligned}
D & \left(S_{m}^{t}(x), S_{n}^{t}(x)\right) \\
& =\max \left\{\sup _{t \in A} \sup _{\lambda \in[0,1]}\left|\sum_{k=n+1}^{m} f_{k}(t)_{\lambda}^{-}\right|, \sup _{t \in A} \sup _{\lambda \in[0,1]}\left|\sum_{k=n+1}^{m} f_{k}(t)_{\lambda}^{+}\right|\right\} \\
& \leq \sum_{k=n+1}^{m} \max \left\{\sup _{t \in A} \sup _{\lambda \in[0,1]}\left|\left(f_{k}^{t}\right)^{-}(\lambda)\right|, \sup _{t \in A} \sup _{\lambda \in[0,1]}\left|\left(f_{k}^{t}\right)^{+}(\lambda)\right|\right\} \\
& \leq \sum_{k=n+1}^{m} M_{k}<\epsilon .
\end{aligned}
$$

Therefore, by the Cauchy criterion in Corollary 28, the series ${ }_{\oplus} \sum_{k} f_{k}^{t}(x)$ converges uniformly on $A$.

We may now give the following, which is the main for some applications of power series of fuzzy numbers (see [22]). We may state a condition under which term-by-term $\mathrm{H}$ differentiation of an infinite series is allowable.

Definition 31. Let $x$ be any element and $x_{0}$ the fixed element. Then, the power series with fuzzy coefficients $a_{n}$ is in the form

$$
\begin{gathered}
\sum_{n=0}^{\infty} a_{n}\left(x-x_{0}\right)^{n}=a_{0} \oplus a_{1}\left(x-x_{0}\right) \oplus a_{2}\left(x-x_{0}\right)^{2} \\
\oplus \cdots \oplus a_{n}\left(x-x_{0}\right)^{n} \oplus \cdots
\end{gathered}
$$

and the radius of convergence $R$ is defined by

$$
R:=\lim _{n \rightarrow \infty} \frac{a_{n}^{+}(0)}{a_{n+1}^{+}(0)}
$$

which is also given provided that the limit on the right hand side exists, where $0 \leq R \leq \infty$.

Definition 32. Suppose a power series ${ }_{\oplus} \sum_{n=1}^{\infty} a_{n}\left(x-x_{0}\right)^{n}$ with radius of convergence $R$. Then, the set of the points from an interval at which the series is convergent is called the interval of convergence such that $\left|x-x_{0}\right|<R \Leftrightarrow x \in\left(x_{0}-R, x_{0}+R\right)$ which must be either $\left.] x_{0}-R, x_{0}+R[,] x_{0}-R, x_{0}+R\right],\left[x_{0}-\right.$ $R, x_{0}+R\left[\right.$, or $\left[x_{0}-R, x_{0}+R\right]$.

Theorem 33. A power series with fuzzy coefficient $s_{\oplus} \sum_{n \geq 0} a_{n} x^{n}$ and the $n$-fold derived series defined by

$$
\sum_{n \geq k} n(n-1) \cdots(n-k+1) a_{n} x^{n-k}
$$

have the same radius of convergence.

Theorem 34 (cf. [23]). If $\sum_{\oplus \geq 0} a_{n} x^{n}$ has radius of convergence $R>0$, then $f^{t}(x)={ }_{\oplus} \sum_{n \geq 0} a_{n} x^{n}$ is H-differentiable in $|x|<R$ and $\left(f^{t}\right)^{\prime}(x)={ }_{\oplus} \sum_{n \geq 1} n a_{n} x^{n-1}$. Consequently, $\left(f^{t}\right)^{(n)}(x)$ exists for every $n \geq 1$ and every $x$ with $|x|<R$, and

$$
\left(f^{t}\right)^{(n)}(x)=\sum_{\oplus}^{\infty} n(n-1) \cdots(n-k+1) a_{n} x^{n-k},
$$

$(|x|<R)$.

The fuzzy coefficients $a_{n}$ are uniquely determined, and

$$
\begin{aligned}
a_{n} & =\left[\left(a_{n}\right)_{\lambda}^{-},\left(a_{n}\right)_{\lambda}^{+}\right] \\
& =\left[\frac{\left(f^{t}\right)^{(n)}(0)}{n !}\right]_{\lambda}=\left[\frac{\left(\left(f^{0}\right)^{(n)}\right)_{\lambda}^{-}}{n !}, \frac{\left(\left(f^{0}\right)^{(n)}\right)_{\lambda}^{+}}{n !}\right] .
\end{aligned}
$$

Proof. Let

$$
\begin{aligned}
f^{t}(x) & =\sum_{\oplus}^{\infty} a_{n=0} x^{n} \\
& =\left[\sum_{n=0}^{\infty}\left(a_{n}\right)_{\lambda}^{-} t^{n}, \sum_{n=0}^{\infty}\left(a_{n}\right)_{\lambda}^{+} t^{n}\right] \in E^{1}
\end{aligned}
$$

have radius of convergence $R>0$. We have to show the existence of H-differentiable $\left(f^{t}\right)^{\prime}(x)$ in $|t|<R$ and that $\left(f^{t}\right)^{\prime}$ is of the stated form. By Theorem 33 with $k=1$, the derived series $_{\oplus} \sum_{n \geq 1} n a_{n} x^{n-1}$ converges for $|t|<R$ and defines a fuzzy-valued function, say $g^{t}(x)$, in $|t|<R$. We show that $\left(f^{t}\right)^{\prime}(x)=g^{t}(x)$ for all $x, t \in(-R, R)$.

Let $t \in(-R, R)$ be fixed. Then take a positive $r<R$ such that $|t|<r$; that is, $r=(R+|t|) / 2$. Also, let $h \in \mathbb{R}$ with 
$0<|h|<(R-|t|) / 2$. We have $|t+h| \leq|t|+|h|<|t|+(R-|t|) / 2=$ $(R+|t|) / 2=r$. We consider

$$
\begin{gathered}
D\left(\frac{f^{t}(x+h) \ominus f^{t}(x)}{h}, g^{t}(x)\right) \\
=\left[\frac{f_{\lambda}^{-}(t+h)-f_{\lambda}^{-}(t)}{h}-g_{\lambda}^{-}(t),\right. \\
\left.\frac{f_{\lambda}^{+}(t+h)-f_{\lambda}^{+}(t)}{h}-g_{\lambda}^{+}(t)\right]_{\lambda} \\
=\sum_{\oplus} \sum_{n \geq 2} a_{n}\left(\frac{(t+h)^{n}-t^{n}}{h}-n t^{n-1}\right),
\end{gathered}
$$

where $\max \{|t|,|t+h|\} \leq r<R$. By taking into account Taylors theorem on the interval with endpoints, we get

$$
\left|\frac{(t+h)^{n}-t^{n}}{h}-n t^{n-1}\right| \leq|h| \frac{n(n-1)}{2} r^{n-2} .
$$

Therefore we have $h \rightarrow 0$,

$$
\begin{aligned}
& D\left(\left(f^{t}\right)^{\prime}(x), g^{t}(x)\right) \\
& \quad=D\left(\sum_{\oplus \geq 2} a_{n}\left(\frac{(t+h)^{n}-t^{n}}{h}-n t^{n-1}\right), \overline{0}\right) \\
& \quad \leq \sup _{\lambda \in[0,1]} \max \left\{\left|\frac{h}{2} \sum_{n \geq 2}\left(a_{n}\right)_{\lambda}^{-} n(n-1) r^{n-2}\right|,\right. \\
& \left.\left|\frac{h}{2} \sum_{n \geq 2}\left(a_{n}\right)_{\lambda}^{+} n(n-1) r^{n-2}\right|\right\} \rightarrow 0 .
\end{aligned}
$$

Consequently, by (57), it follows that $\left(f^{t}\right)^{\prime}(x)$ exists and equals $g^{t}(x)$. Since $x$ is arbitrary, this holds at any interior point in $|x|<R$. On the other hand, this argument gives that all the $\mathrm{H}$-derivatives exist in $|x|<R$.

Corollary 35. Consider $\left\{f_{n}^{t}\right\}$ is a sequence of fuzzy-valued functions. Then the following hold:

(i) $\left\{f_{n}^{t}\right\} \in C_{F}^{1}[a, b]$ (H-differentiable continuous fuzzyvalued function);

(ii) there exists a point $x_{0} \in[a, b]$ such that ${ }_{\oplus} \sum_{k} f_{k}^{t}\left(x_{0}\right)$ converges;

(iii) $\sum_{\oplus}\left(f_{k}^{t}\right)^{\prime}$ converges uniformly on $[a, b]$.

Then $\sum_{\oplus} \sum_{k} f_{k}^{t}$ converges uniformly on $[a, b]$ to a H-differentiable fuzzy-valued function $F$,

$$
F^{\prime}(x)=\left(\sum_{\oplus} f_{k}^{t}(x)\right)^{\prime}=\sum_{\oplus} \sum_{k}\left(f_{k}^{t}(x)\right)^{\prime}
$$

for all $x, t \in[a, b]$ and $\lambda \in[0,1]$.

\section{Conflict of Interests}

The authors declare that there is no conflict of interests regarding the publication of this paper.

\section{Acknowledgment}

The authors record their pleasure to the anonymous referee for his/her constructive report and many helpful suggestions on the main results.

\section{References}

[1] H. N. Jahnke, The Foundation of Analysis in the 19th Century: Weierstrass. A History of Analysis, AMS Bookstore, 2003.

[2] L. Imre, Proofs and Refutations, Cambridge University Press, 1976.

[3] M. Başarir, "On some new sequence spaces of fuzzy numbers," Indian Journal of Pure and Applied Mathematics, vol. 34, no. 9, pp. 1351-1357, 2003.

[4] O. Talo and F. Başar, "On the space $b v_{p}(F)$ of sequences of $p$ bounded variation of fuzzy numbers," Acta Mathematica Sinica, English Series, vol. 24, no. 7, pp. 965-972, 2008.

[5] F. Başar and B. Altay, "On the space of sequences of $p$ bounded variation and related matrix mappings," Ukrainian Mathematical Journal, vol. 24, no. 1, pp. 136-147, 2003.

[6] U. Kadak and M. Ozluk, "Some new sets of sequences of fuzzy numbers with respect to the partial metric," The Scientific World Journal, vol. 2014, Article ID 735703, 11 pages, 2014.

[7] L. A. Zadeh, “Fuzzy sets," Information and Computation, vol. 8, pp. 338-353, 1965.

[8] R. Goetschel and W. Voxman, "Elementary fuzzy calculus," Fuzzy Sets and Systems, vol. 18, no. 1, pp. 31-43, 1986.

[9] K. H. Lee, First Course on Fuzzy Theory and Applications, Springer, Berlin, Germany, 2005.

[10] P. Diamond and P. Kloeden, "Metric spaces of fuzzy sets," Fuzzy Sets and Systems, vol. 35, no. 2, pp. 241-249, 1990.

[11] S. Nanda, "On sequences of fuzzy numbers," Fuzzy Sets and Systems, vol. 33, no. 1, pp. 123-126, 1989.

[12] B. Bede and S. G. Gal, "Almost periodic fuzzy-number-valued functions," Fuzzy Sets and Systems, vol. 147, no. 3, pp. 385-403, 2004.

[13] M. L. Puri and D. A. Ralescu, "Differentials of fuzzy functions," Journal of Mathematical Analysis and Applications, vol. 91, no. 2, pp. 552-558, 1983.

[14] O. Talo and F. Başar, "Determination of the duals of classical sets of sequences of fuzzy numbers and related matrix transformations," Computers \& Mathematics with Applications, vol. 58, no. 4, pp. 717-733, 2009.

[15] L. Stefanini, "A generalization of Hukuhara difference for interval and fuzzy arithmetic," in Soft Methods for Handling Variability and Imprecision, vol. 48 of Series on Advances in Soft Computing, 2008.

[16] L. Stefanini and B. Bede, "Generalized Hukuhara differentiability of interval-valued functions and interval differential equations," Nonlinear Analysis. Theory, Methods \& Applications, vol. 71, no. 3-4, pp. 1311-1328, 2009.

[17] U. Kadak and F. Başar, "On some sets of fuzzy-valued sequences with the level sets," Contemporary Analysis and Applied Mathematics, vol. 1, no. 2, pp. 70-90, 2013.

[18] U. Kadak, "On the sets of fuzzy-valued function with the level sets," Journal of Fuzzy Set Valued Analysis, vol. 2013, Article ID 00171, 13 pages, 2013.

[19] M. Hukuhara, "Intégration des applications mesurables dont la valeur est un compact convex," Funkcialaj Ekvacioj, vol. 10, pp. 205-229, 1967. 
[20] U. Kadak and F. Başar, "On fourier series of fuzzy-valued functions," The Scientific World Journal, vol. 2014, Article ID 782652, 13 pages, 2014.

[21] G. A. Anastassiou, Fuzzy Mathematics: Approximation Theory, Studies in Fuzziness and Soft Computing, 251, 2010.

[22] U. Kadak and F. Başar, "Power series of fuzzy numbers," AIP Conference Proceedings, vol. 1309, pp. 538-550, 2010.

[23] S. Ponnusamy, Foundations of Mathematical Analysis, Springer, 2010. 


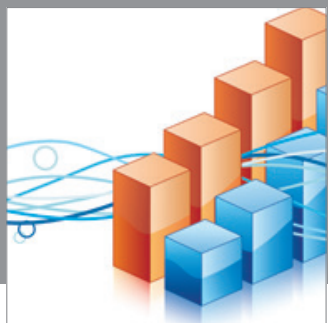

Advances in

Operations Research

mansans

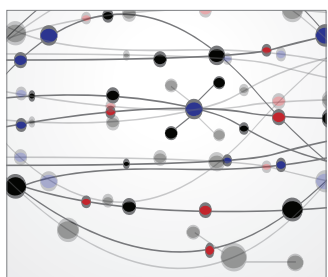

The Scientific World Journal
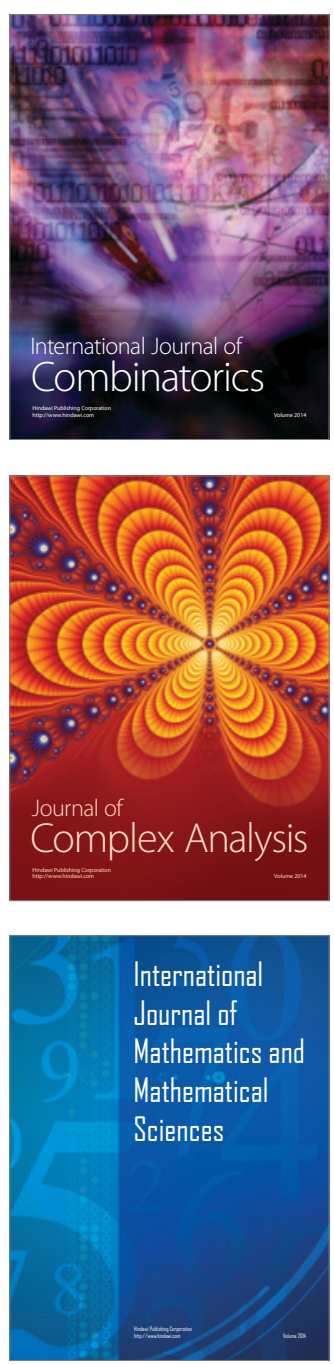
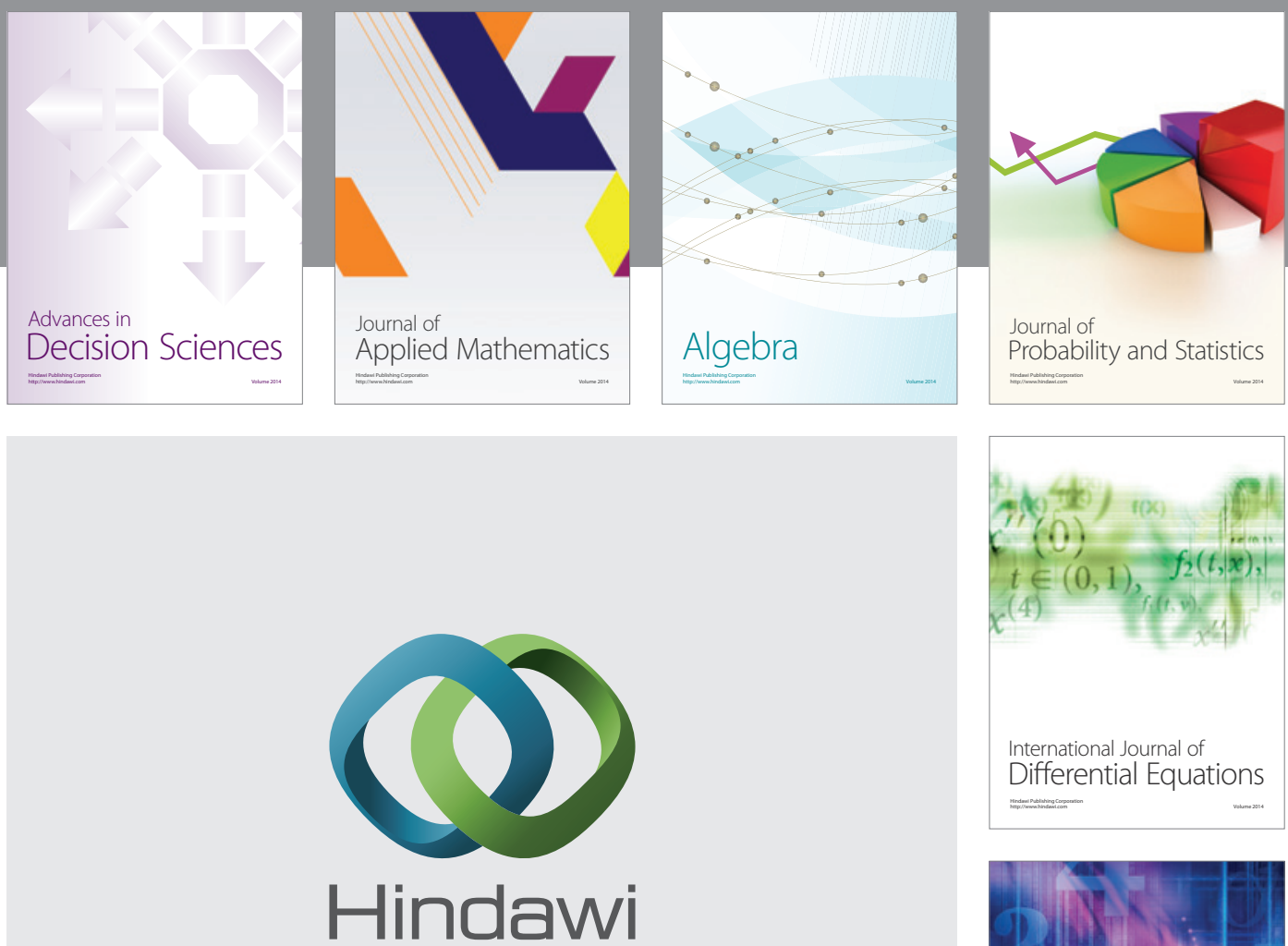

Submit your manuscripts at http://www.hindawi.com
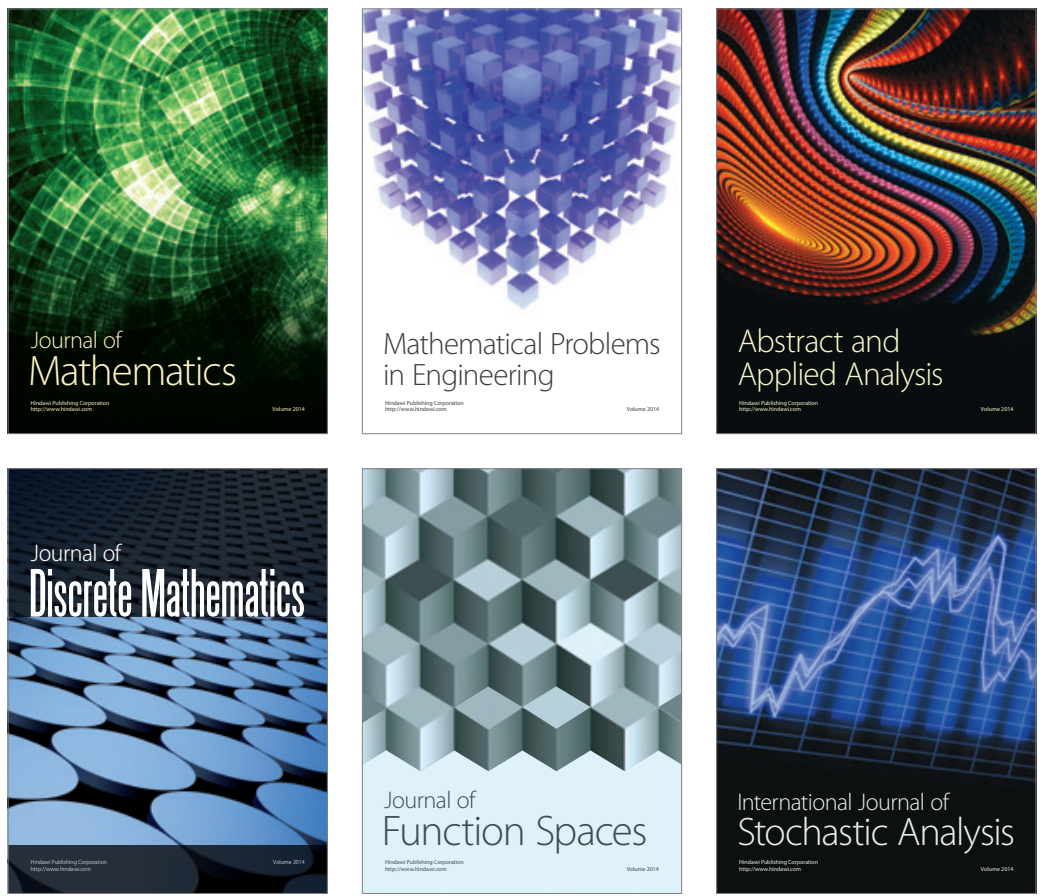

Journal of

Function Spaces

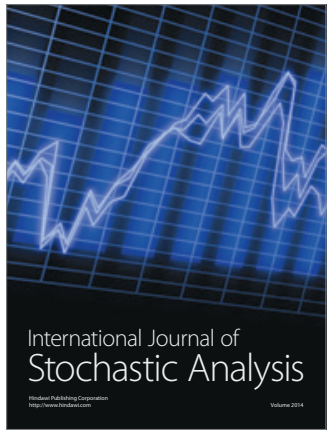

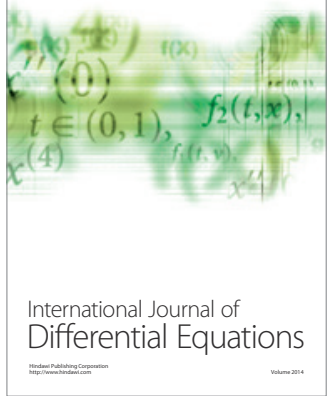
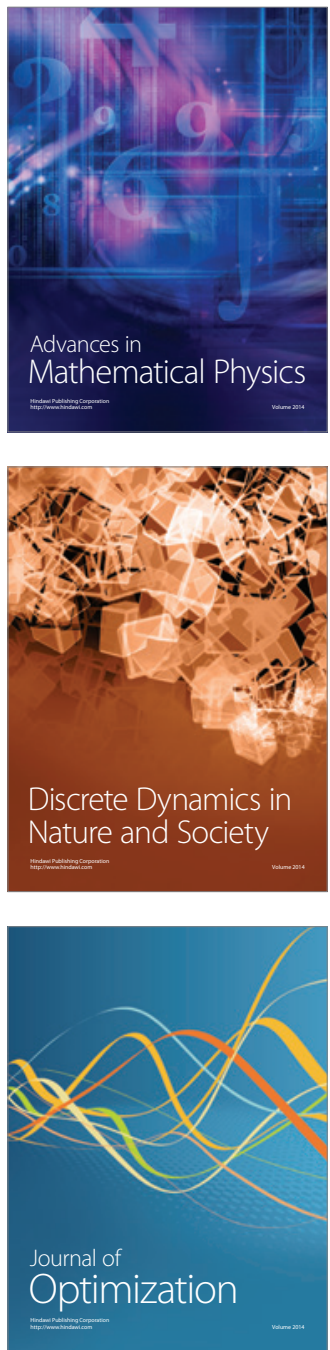